\title{
PATHOLOGY/BIOLOGY
}

\author{
LokMan Sung, ${ }^{1,2}$ M.D.; Kilak Kesha, ${ }^{3}$ M.D.; Sarah Avedschmidt, ${ }^{2}$ M.D.; Kelly Root,${ }^{1}$; and \\ Leigh Hlavaty, ${ }^{1,2}$ M.D.
}

\section{The Modern Compound Bow}

\begin{abstract}
Bows and arrows are ancient weapons that have risen and fallen as the preeminent armaments used by man. Because of the ubiquity of firearms, fatalities from archery injuries in the United States have radically declined. However, when deaths involving this weapon do present themselves, the paucity of reference materials can be a hurdle for forensic pathologists and other forensic scientists. This article will provide a brief history of the origins of the bow and the inception of the compound bow. Comparing and contrasting the structures comprising a traditional bow to those of the modern compound bow will provide insight into how these components function in unison to propel arrows.
\end{abstract}

KEYWORDS: forensic science, compound bow, arrow, bow sight, mechanical release, forensic pathology

The bow and arrow is sometimes forgotten as an invention that forever changed mankind's course in history. In the Modern Era, people living in industrialized societies rarely contemplate the large time and energy expenditure that early humans dedicated to food gathering. By increasing wounding through storing potential energy in a bow and using arrows fit with cutting edges, humans could shift valuable resources to other endeavors. There is much debate over the exact impact this weapon has had on the primitive human's cognitive and social advancement; however, scholars agree that combining the individual components, such as wood, bones, plants, tendons, and stone, into this complex projectile technology required significant intellectual innovation (1).

Archaeologists have recently increased their search for this weapon's origins, but have met many obstacles. The central difficulty in discovering artifacts is the relative lack of preserved materials for study. The organic components used in the bow's construction, such as tree limbs, bones, and plant material, deteriorated over time. The arrows were also formed predominantly from labile materials such as wood and feathers. Only the knapped arrowheads stood a greater chance against time's slow destruction. To date, the oldest indications of the bow and stone-tipped arrow use have come from Sibudu Cave, KwaZulu-Natal, South Africa, during the Pleistocene period, some 64,000 years ago (2).

Equally difficult is identifying the eras where major alterations to the bow's design and fabrication occurred. At its most fundamental level, the original bow needed only two components, a flexible tree limb and a bowstring. A supple tree limb or curved tree branch bent into a single D-shaped arc had a plant fiber bowstring strung, or "braced," onto its tips (3). The operator's bow hand grasped the mid-portion of the limb and the dominant

\footnotetext{
${ }^{1}$ Wayne County Medical Examiner's Office, Detroit, MI.

${ }^{2}$ Department of Pathology, University of Michigan, Ann Arbor, MI.

${ }^{3}$ Department of Forensic Pathology, National Forensic Pathology Service of New Zealand, Auckland, New Zealand.

Received 6 Dec. 2016; and in revised form 5 Jan. 2017; accepted 6 Mar. 2017.
}

drawing hand pulled the bowstring back. When in the braced state and throughout the entire draw cycle, the distal ends of the limbs faced the user, and at no point did the bowstring touch the limbs other than at the tips (Fig. 1A). The famous English longbow epitomized the retained primal bow shape but reflected alterations to the bow's construction materials. A trimmed rod of wood, or "stave," from the Yew tree (Taxus baccata) replaced intact tree limbs, where the stave's back, the edge opposite the bowstring, was the outer layer, or sapwood, of the tree, and the belly, the edge facing the bowstring, was the inner rings, or heartwood. This proved ideal because the heartwood could withstand the considerable compression stress while the sapwood resisted the tension stress created when drawing the bow (4).

Many of bows in Asia veered from the longbow's single arc design to the undulating shape now known as the recurve bow (Fig. 1B). This bow's mid-portion still retains the arc but the limb tips point away from the archer, and the bowstring rests against the limb's distal portions when braced with a bowstring. These Asiatic bows consisted of wood, horn, and sinew, and the portions touching the string were rigid and did not flex upon drawing. These static-recurve bow limbs contrast with many contemporary working-recurve bows where the limb portions touching the bowstring do flex when drawn (5).

Furthermore, the construction materials of bows create the dichotomies of "self bows" and "composite" bows. Like the traditional English longbow, the structure of the self bow originates from a single tree. As self bows represent simplicity, composite bows are very much the opposite. The initial composite bows, akin to Asiatic recurve bows, bond together animal horns, sinew, and wood with complex glues to optimize tensile and compression strength. The latest composite bows integrate aluminum, fiberglass, and carbon fiber.

Another level of complexity comes from the "compound" bow. Compound bows and composite bows are frequently mistaken due to their similar sounding names. All compound bows are composite but the opposite is not true. Currently, composite describes the materials and methods, while compound reflects the design. 
A

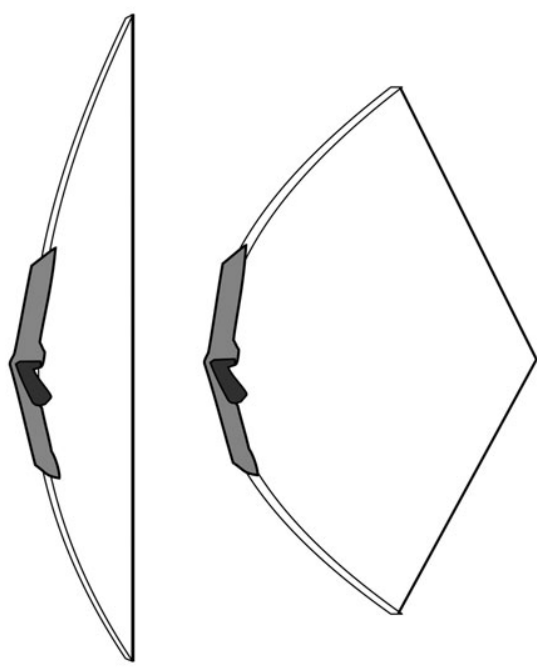

B

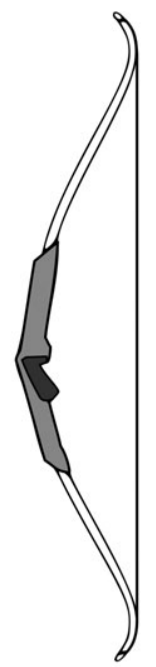

At rest

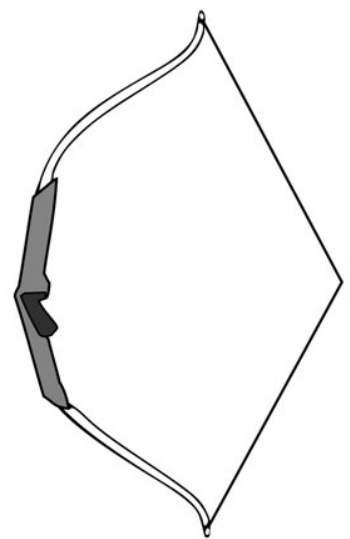

Drawn

FIG. 1-Basic bow design: (A) longbow; (B) recurve bow.

Holless Wilbur Allen invented the compound bow in 1966. The driving force came from an unsuccessful hunting excursion in the Fall of 1965, where Mr. Allen sought to create a bow that could increase arrow velocity (6). Because decreased arrow mass only marginally increased velocity, focus shifted to discovering ways of storing greater potential energy. The solution came from the mechanical advantage gained through pulley wheels or cams mounted at the limb ends. With both the longbow and recurve bow, the force required to draw the bow increased with limb flexion, and these traditional bows required considerable force to sustain the anchored position (holding weight). On the other hand, the compound bow allows one to reach a peak force at the pulley wheel's apex and then pivots to steadily decrease the holding weight. The decreased holding weight requires less effort to maintain the full draw and provides more allowances to aim and shoot. These changes revolutionized archery and launched the compound bow into prominence.

The summary purpose of the compound bow is to provide a mechanism to propel an arrow at an intended target with greater force. While the concept is straightforward, extensive research and development has been spent in shaping the modern compound bow. This study provides forensic pathologists and

scientists with a background to the key components of this complex weapon and will facilitate an understanding of the technical role of those components in the function of the compound bow.

\section{Components}

Deconstruction of the compound bow into its constituent parts will allow for the most straightforward description and illustration of how those parts contribute to the operation of the bow. The two categories that these components fall into are those intrinsic to the weapon and common extrinsic accessories that greatly improve the function of the compound bow.

\section{Intrinsic}

Intrinsic components are those required to operate the compound bow; without them the weapon would be useless or unsafe to shoot. The most critical intrinsic components for this discussion include the riser, arrow shelf, grip, limbs, cams, and bowstring (Fig. 2).

Riser-The "riser" is the main portion of a compound bow and contains or attaches to most of the intrinsic and extrinsic components. It is the most substantial component of the bow, is either right-handed or left-handed, and comprises much of the

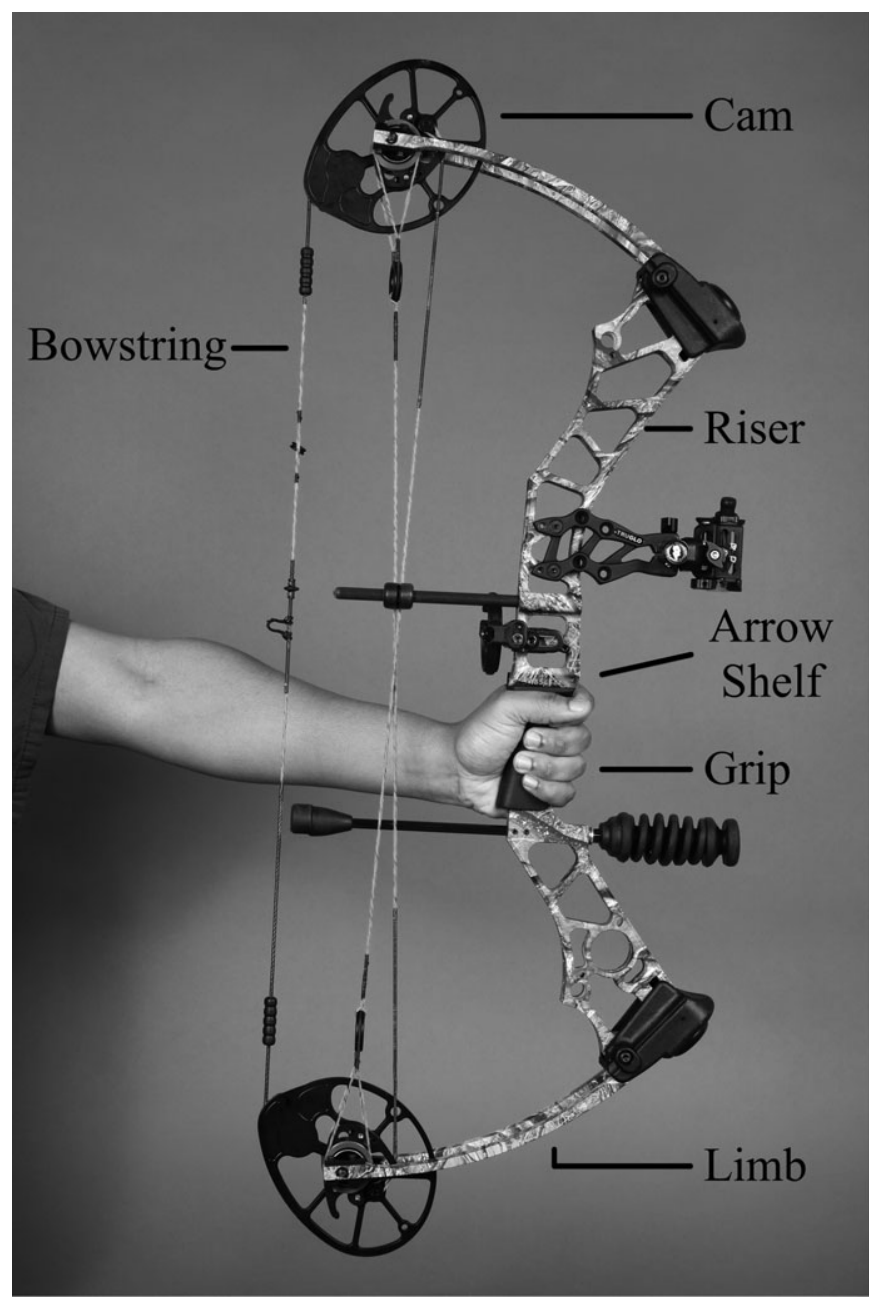

FIG. 2-Modern compound bow highlighting intrinsic components. 
bow's weight. Some risers are solid pieces (Fig. 3A), while others aim for weight reduction by skeletonizing the riser with cutouts (Fig. 3B).

The original riser by Allen was of simple pine boards bonded by glue, and for decades, wood played a prominent place in compound bow construction (6). Today, risers are most commonly manufactured from metal or metal alloys such as aluminum, and magnesium. In its construction, the riser can be cast from molds, extruded through a die, or forged and machined from a single piece of metal. Although the cost is prohibitive for many current consumers, a few manufactures integrate or solely construct risers from carbon fiber. Carbon fiber usage reflects a natural progression of technology because of its increased strength and negligible weight. An even smaller niche market driven by private ingenuity is the creation of polymer risers through 3D printing (7).

There are exhaustive arguments over the ease of use or forgiveness to human inconsistencies of one particular bow compared to another. Three areas with consistent discussion are the architecture, "axle-to-axle length," and "brace height" of the risers (Fig. 4). These variables can be mutually exclusive, but frequently all affect each other in the performance of the bow. In regard to the architectural style, risers are either deflex, inline, or reflex (8). These divisions compare the location of the "limb pockets," where the limbs attach to the riser, to the "grip," where the bow hand grasps the weapon (further discussion below). If the limb pockets are anterior to the grip, the riser is deflex. If the limb pockets are along the same axis as the grip, then the riser is inline. Reflex risers have the limb pockets positioned posterior to the grip. The axle-to-axle length takes into account the riser together with the limbs. The distal ends of the limbs hold the "cams" of the bow with a pin or rod (further discussion below).

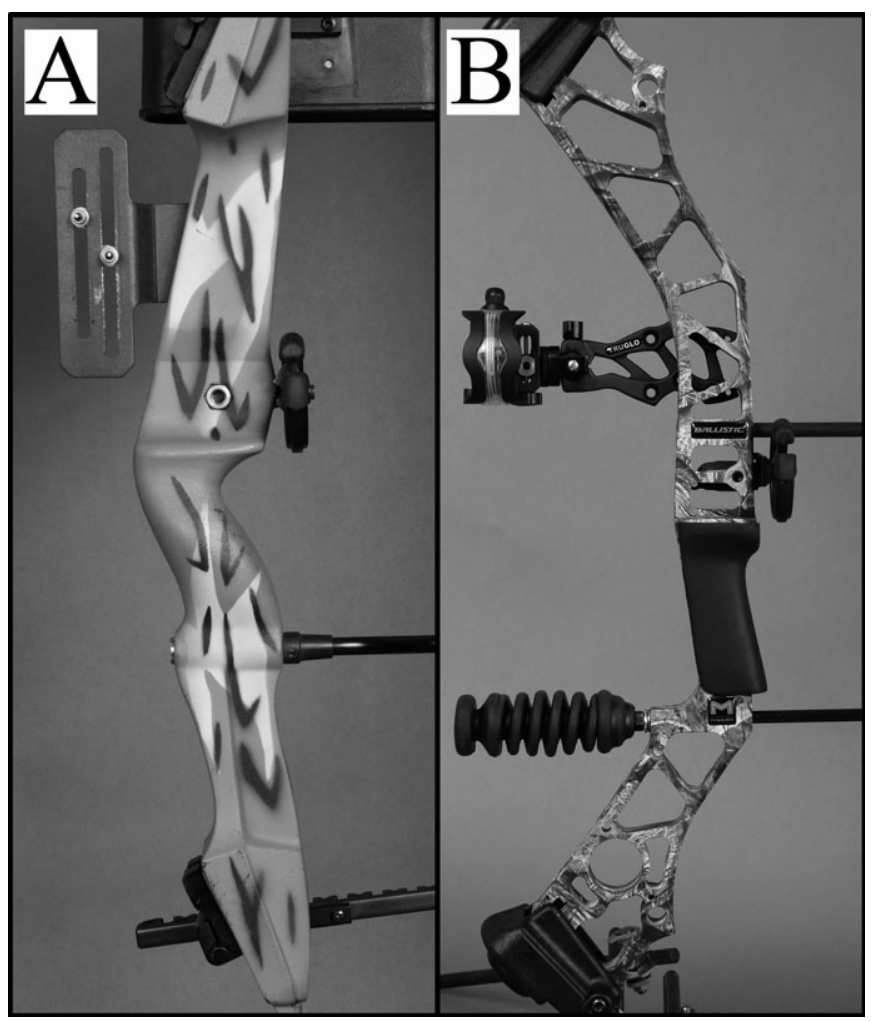

FIG. 3-Compound bow risers: (A) solid riser; (B) skeletonized riser.
The distance between the rods is the axle-to-axle length. The brace height describes the distance of the bowstring to the grip.

The closer the bowstring is placed to the grip, such as in reflex risers and lower brace heights, the further the bowstring needs to be pulled in order to obtain full draw, otherwise known as having a longer "draw length." Bows with greater draw lengths generally have increased ability to store potential energy, which allows increased velocities of arrows. A drawback is that the bow responds greater to operator error than those with deflex risers and higher brace heights. All things being equal, bows with greater axle-to-axle lengths allow for greater draw lengths. However, bows with greater axle-to-axle lengths can be more difficult to maneuver. An archer must select the bow appropriate for their arms and draw length because each compound bow is configured to a specific draw length.

Arrow Shelf-The "arrow shelf" serves as a place to hold the arrow on the bow, preparing for its firing (Fig. 5A). Many traditional (noncompound) bows do not have arrow shelves and the arrow rests on top of the archer's bow hand through the operational cycle of drawing, aiming, and firing. This method of arrow constraint is inconsistent and increases the potential for shot-to-shot variance. The integrated arrow shelf provides the archer with a defined area for the arrow to sit and helps to reduce some human variability.

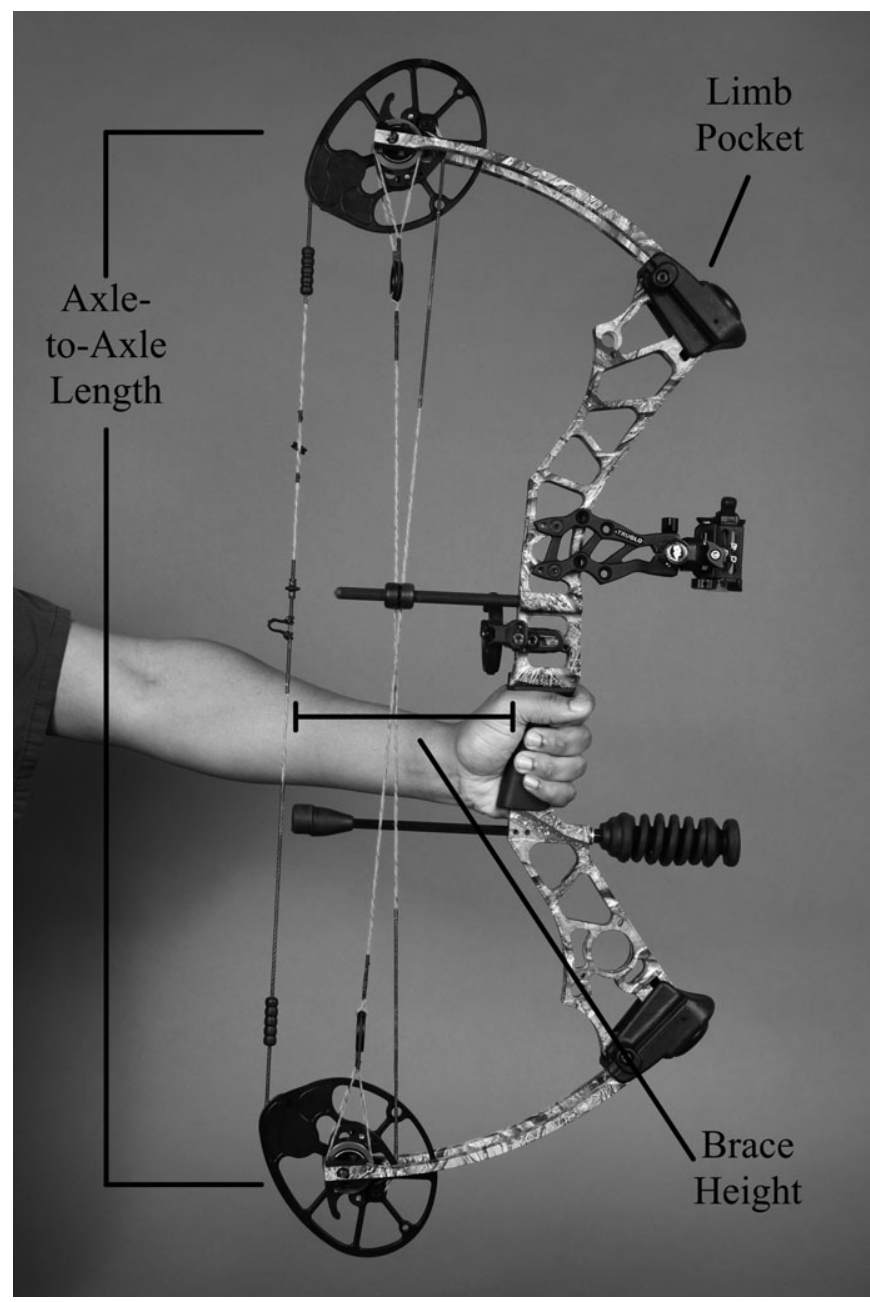

FIG. 4-Modern compound bow highlighting architectural design. 
An unintended consequence of the arrow shelf is the production of noise from the friction between the shelf and arrow with the drawing of the bow. Affixing materials such as moleskin, leather, calf hair, felt, rubber, and tape are some of the things archers use for combating the noise.

Most compound bows have an "arrow rest" (further discussion below) which takes up most of the function of the arrow shelf. However, the position of the arrow shelf on the riser serves an even more important function than just a place to balance the arrow. The arrow shelves of many modern compound bows are not flush with the face of the riser, but are "center-shot" or offset to place the arrow in the center of the riser and bowstring. By having all three aligned, the kinetic energy released from the limbs conducts directly into the arrow along a single vector and minimizes energy wasted by even the slightest deviation (Fig. $5 B$ ).

Grip-Typically, the archer interfaces with only two or three parts of the compound bow. Of those, the grip is the most substantial. It comprises the mid-portion of the riser directly under the arrow shelf. As with the other portions of the riser, the grip of the compound bow is not ambidextrous and the bow hand of the archer grasps this area. The grip can either be permanently molded to fit a generic hand (Fig. 6A) or made of replaceable material for detailed individualization (Fig. 6B, $C$ ).

With regard to the hand placement on the grip, naive shooters may incorrectly translate principles indoctrinated for proper handgun grip formation to the compound bow. In handguns, the shooter uses both hands to firmly envelop the firearm grip in a $360^{\circ}$ fashion. This allows the shooter to put equal force on all aspects of the firearm grip and control recoil while firing multiple rounds. A two-handed grip is not possible with the bow. If grasped firmly, the single hand creates an imbalance of forces that places torque on the entire bow and introduces errors in accuracy and precision. Archers therefore adopt a barely present grip. Drawing the bow presses the posterior portion of the grip into the webbing between the first and second fingers and the thenar eminence of the bow hand. This pressure alone will keep the bow in place, negating the need to actually grasp the grip.

A bow "wrist sling" can further decrease the archer's hold on the grip. When minimal strength grips the bow, the weapon may fall from the hand and onto the floor upon release of the arrow and eliminate the pressure keeping the bow in the hand.
Knowing the bow may potentially fall, and some archers unconsciously grasp the grip on release of the arrow and inadvertently torque the bow, creating inconsistent shooting. The wrist sling attaches to the riser and gently encircles the wrist of the bow hand. After firing the arrow, the wrist sling keeps the bow in the hand and provides the psychological safety net some archers desire.

Limb-The limbs are the flexible portions of the bow and act as cantilever springs storing the potential energy imparted by the archer. The limbs are multiple laminated layers of fiberglass bonded by resins and glues. The limbs fit into limb pockets at the distal ends of the riser and are threaded with adjustable "limb bolts" (Fig. 7). Tightening the limb bolts pivots the proximal ends of the limbs away from each other and widens the distance between the distal ends of the limbs. This increases the tension on the limbs and bowstring and requires greater force to draw the bow. Each pair of limbs possesses specified rigidity that requires a finite range of force to flex. This range of force is called the draw weight and typically spans $10-15$ pounds. Therefore, one compound bow may have a draw weight between 50 and $60 \mathrm{lbs}$, or 60 and $70 \mathrm{lbs}$, or 70 and $80 \mathrm{lbs}$, for example. For many typical bows, drawing outside of that weight range requires replacing the limbs with ones of increased or decreased stiffness.

The spring force of the limbs propels the arrow forward but also conducts vibrations into the riser. Self bows and early compound bows orient the limbs near vertically (Fig. 8A), and release of the bowstring accelerates the riser forward in the same direction as the arrow. By placing the limbs parallel to each other and perpendicular to the riser, the vibrational spring of the limbs is cancelled, movement of the riser markedly decreases, and noise production falls (Fig. $8 B$ ). Added benefits of parallel limbs are smooth acceleration of the arrow and increased efficiency of energy transfer into the arrow, both of which results in increased arrow velocities.

Cams-The vital components that provide the compound bow with its mechanical advantage over traditional bows are the pulley wheels, or cams, attached to the distal ends of the limbs. The cams are round or oval and are also known as "eccentrics," a name derived from the eccentrically located spokes on which

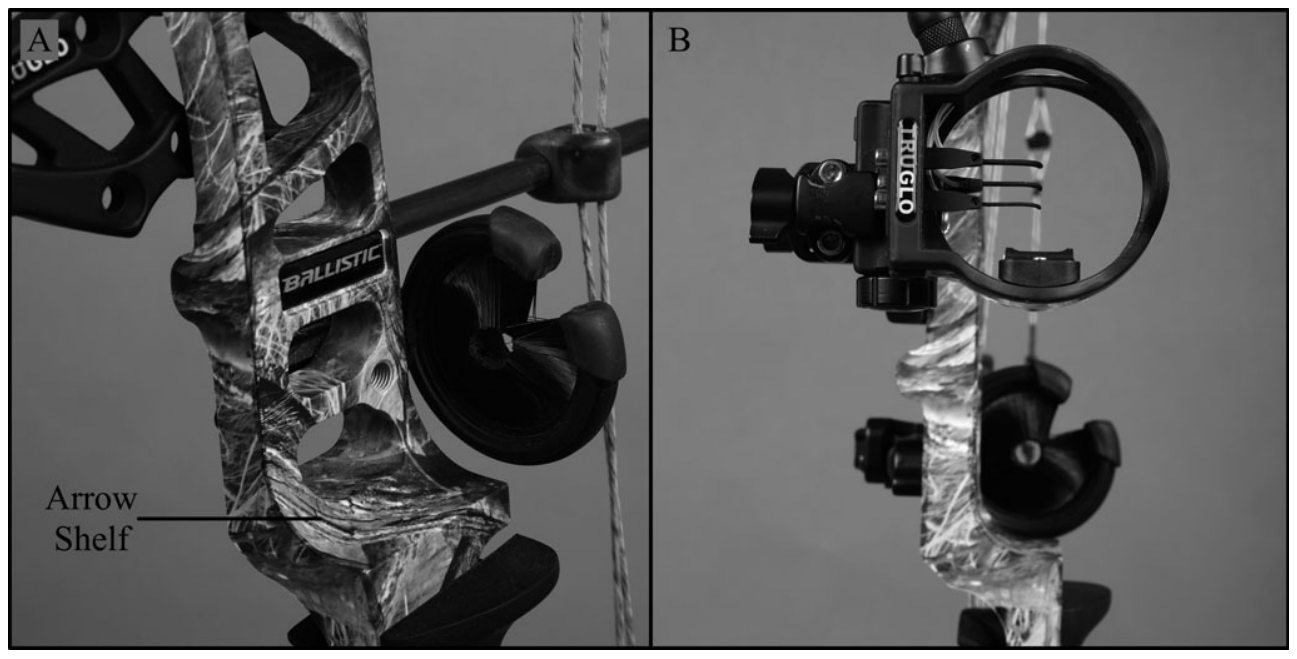

FIG. 5-Arrow shelf: (A) shelf location; (B) center-cut placement of shelf. 


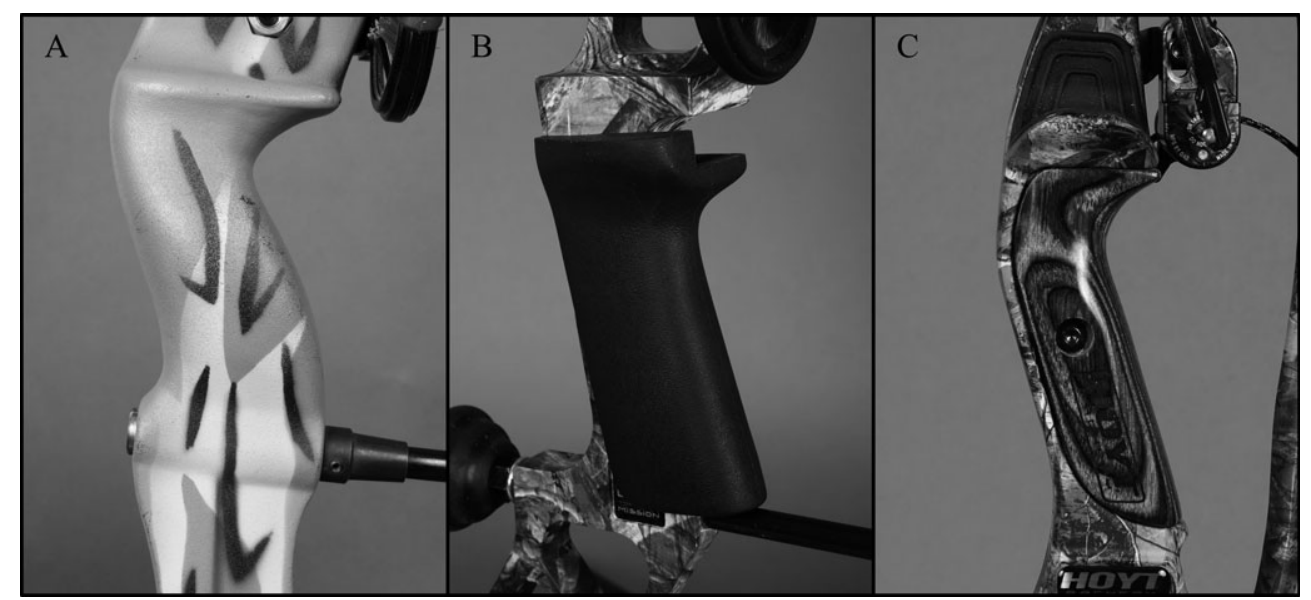

FIG. 6-Grips: (A) molded into riser; (B) replaceable rubber grip; (C) replaceable wood grip.

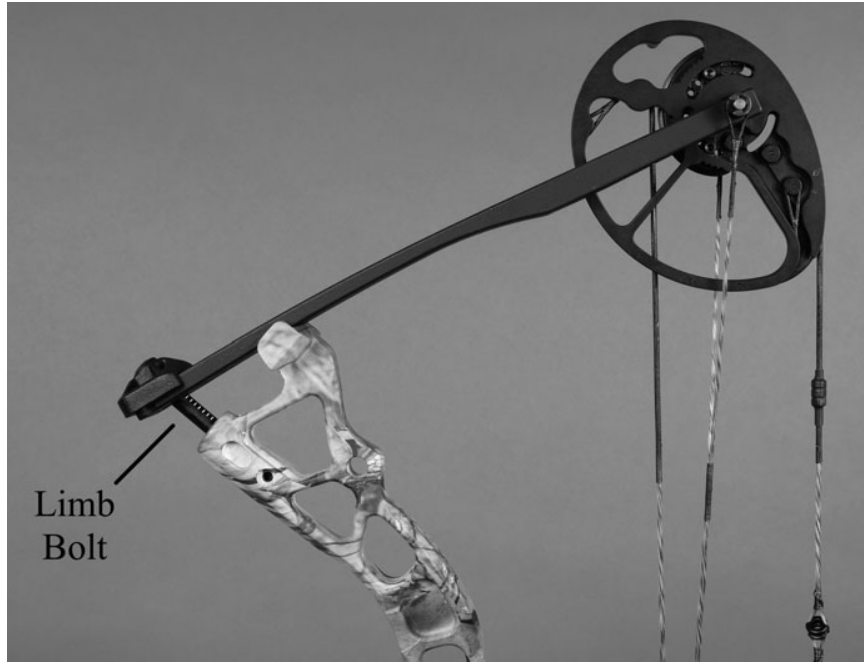

FIG. 7-Bow limb attached with limb bolt.

the cams pivot (Fig. 9). A series of cables and the bowstring tether the cams together and synchronize the turning of these pulley wheels on the drawing of the bow. At the initial stages of the draw, the cables run along the long arc of the wheel and require greater force to turn as the wheel approaches its apex. The apex of cam represents the maximum draw weight, and when the cams pivot and the cables run along the short axis, the force required to complete the remainder of the draw falls to a nadir. The amount of force required to maintain full draw is the holding weight. The percent difference between the maximum draw weight and the holding weight represents the cam's "let off." Commonly, many modern cams provide the archer with let offs of $70 \%$ and $80 \%$ (9). A thorough discussion on the types of cams, the number of cams a compound bow has, and the specifics of the tethering of those cams together is beyond the scope of this review article.

Bowstring-All bows, regardless of the type, require the bowstring to function. Early bowstrings, made of natural materials such as animal gut, animal hair (horse), cotton, flax/linen, tendons, and silk, lose integrity over time and stretch, leaving the string ineffective. Along with wooden risers, natural strings are very sensitive to fluctuations in temperature and humidity.

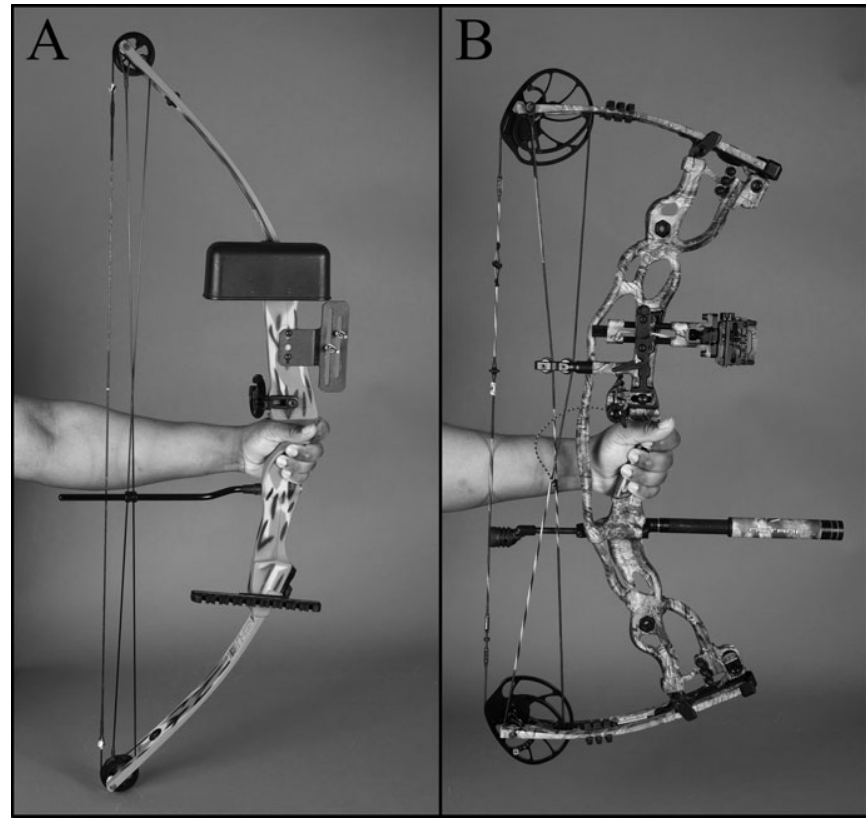

FIG. 8-Limb orientation: (A) near vertical; (B) horizontal.

Synthetic bowstrings resistant to moisture and temperature variations have largely replaced those made of natural materials (10). Early synthetic strings were Dacron polyester. The polyester fiber was more durable than natural fibers but still stretched over time. The subsequent technological leap was the use of the aramid fiber Kevlar. The advantage of Kevlar fiber was its stretch resistance but the major disadvantage was its tendency to break at unpredictable times. A revolution in bowstring construction occurred in the mid-1980s with the introduction of spectra fiber strings. Spectra, along with a similar fiber Dyneema, are made of highly abrasion, chemical, and moisture resistant fibers called ultra-high-modulus polyethylene (UHMPE). These strings proved very reliable and only displayed flaws at extreme high tension and temperatures.

The bowstring is not a single strand of fiber, but multiple strands twisted together with the distal portions wrapped with "serving string" to prevent dividing of the strands. In its production, the strands are under consistent tension to ensure even distribution of force when the bow is strung. The number of twists 


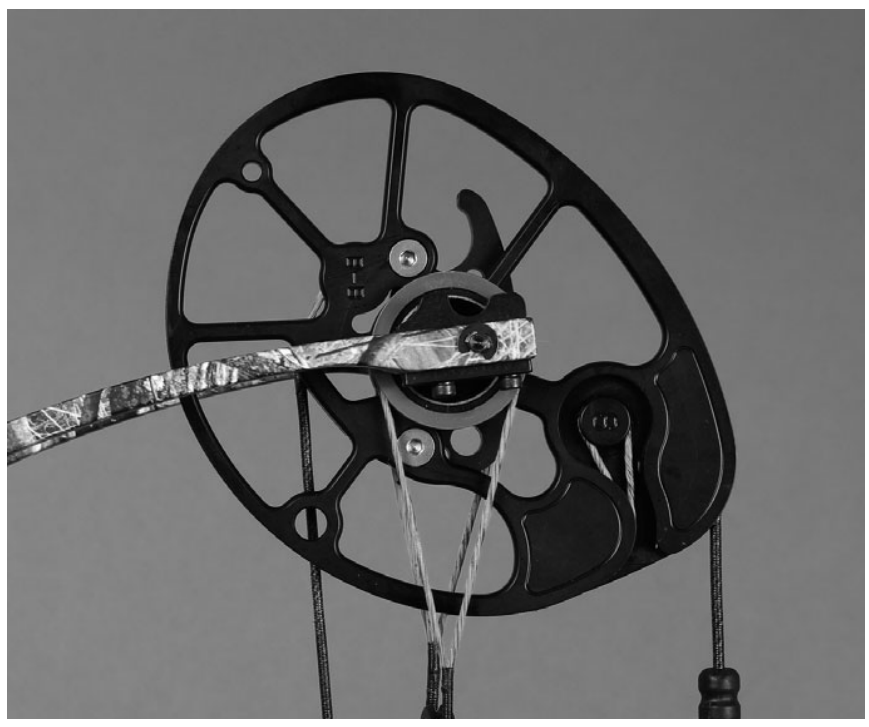

FIG. 9-Example of a bow's cam or eccentric.

a string has also influences string vibration, durability, and the performance of the bow. As with many things, periodic maintenance by applying natural or synthetic waxes will greatly extend the life of the string.

Although not technically part of the bowstring, a "nocking point indicator" is frequently placed onto the string to signal the location that the arrow attaches to it. These points can be brass (Fig. 10A), serving string, or string loops made of cord (Fig. 10B). The purpose is to provide a consistent place for the arrow, which directly translates to shot-to-shot accuracy and precision.

\section{Extrinsic}

The extrinsic components of the compound bow, while not critical or necessary, greatly enhance the performance of the weapon. The discussion focuses on the arrow rest, mechanical release aid, sights, stabilizer, and arrow.

Arrow Rest-In compound bows, the arrow rest compliments the arrow shelf and in many respects supplants the shelf as more functionally significant. Almost all compound bows have arrow rests, and as its name implies, the rest secures the arrow on the bow through the draw cycle and release. The arrow rest housing threads into the riser superior and posterior to the arrow shelf. Excluding specialty arrow rests, such as flipper rests for noncentershot bows and those used for competitive shooting, the most common arrow rests currently used fall into three categories: shoot-through, containment, and drop away arrow rests.

Shoot-through Arrow Rests-The fundamental structure of a shoot-through arrow rest are two spring-loaded prongs separated by a small gap. The arrow fits into the gap, and the prongs support the arrow shaft on the rest, acting as the only contact points with the arrow (Fig. 11A). When released, the arrow travels across the prongs and its friction and forward velocity flex the prongs downward and reduce interference with the arrow's flight. It is critical for the arrow rest to be properly aligned to allow adequate clearance of the arrow and its vanes. Another

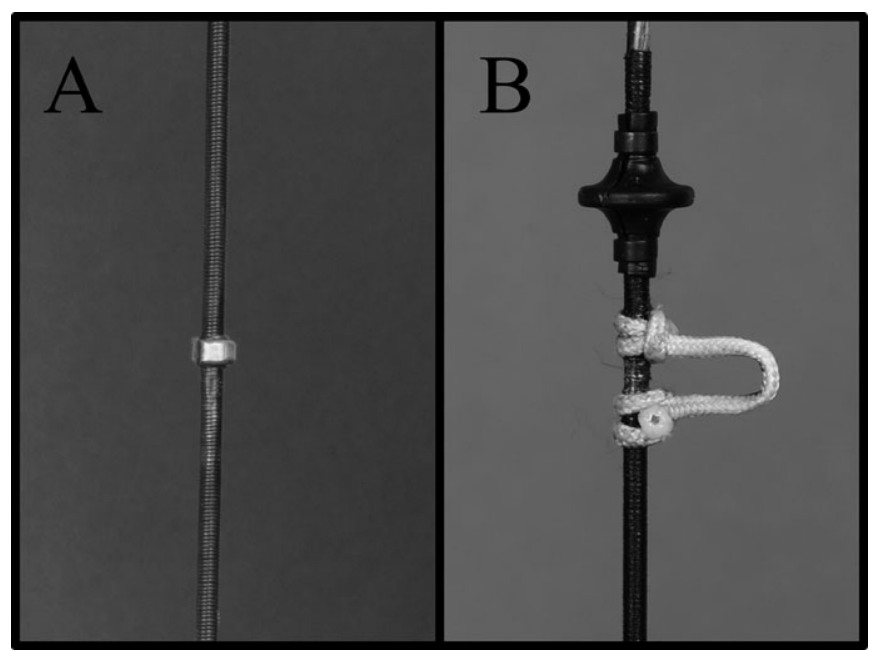

FIG. 10-Bowstring nocking points: (A) brass nock; (B) D-shaped string loop.

consideration with this style of arrow rest is that, because the prongs merely support the arrow, only gravity prevents the arrow from falling off the rest and poor shooting form or sudden movements can dislodge the arrow.

Containment Arrow Rests-Containment arrow rests address the shoot-through arrow rest's lack of ability to capture and prevent arrows from falling off the rest. There are several styles of containment rests, but currently two designs are the most popular. The first are rests that have three, equal distant, points of contact with the arrow shaft (Fig. 11B). The contact points can be brushes, prongs, ramps, or combinations thereof. Clipping, or nocking, the arrow to the string orients the vanes of the arrow to the open intervals of the contact points and will pass through them unperturbed upon release.

The second style of containment rest is the Whisker Biscuit ${ }^{\circledR}$ designed by Trophy Ridge ${ }^{\circledR}$ (Evansville, IN, USA). This arrow rest provides a full $360^{\circ}$ arrow shaft containment by a series of inwardly oriented filaments that extend radially from an outer ring (Fig. 11C). Functionally, the Whisker Biscuit ${ }^{\circledR}$ does provide resistance to most efforts to dislodge the arrow, but this resistance may alter the arrow's flight as the vanes pass through the filaments.

Drop-Away Arrow Rests-Drop-away arrow rests are more mechanically complex than the two previously mentioned rests. The body of the rest houses a spring-loaded axle that extends across the arrow shelf. The proximal end of the axle has a cord that connects to the cables of the bow, and the distal end has a platform, called the launcher (Fig. 11D). In the resting state, the launcher sits on the arrow shelf and the arrow shaft sits on top of the launcher. At full draw of the bow, the cable pulls the cord taut and pivots the launcher into the capture and shooting position. With release of the bowstring, the launcher drops onto the arrow shelf and the arrow flies to the target with complete clearance of the launcher.

Mechanical Release Aid-Archers shooting traditional bows like the longbow and recurve bows typically use the tips of the 2nd, 3rd, and 4th fingers to draw the weapon. Gloves or finger tabs are frequently worn to protect the tips of the fingers from 


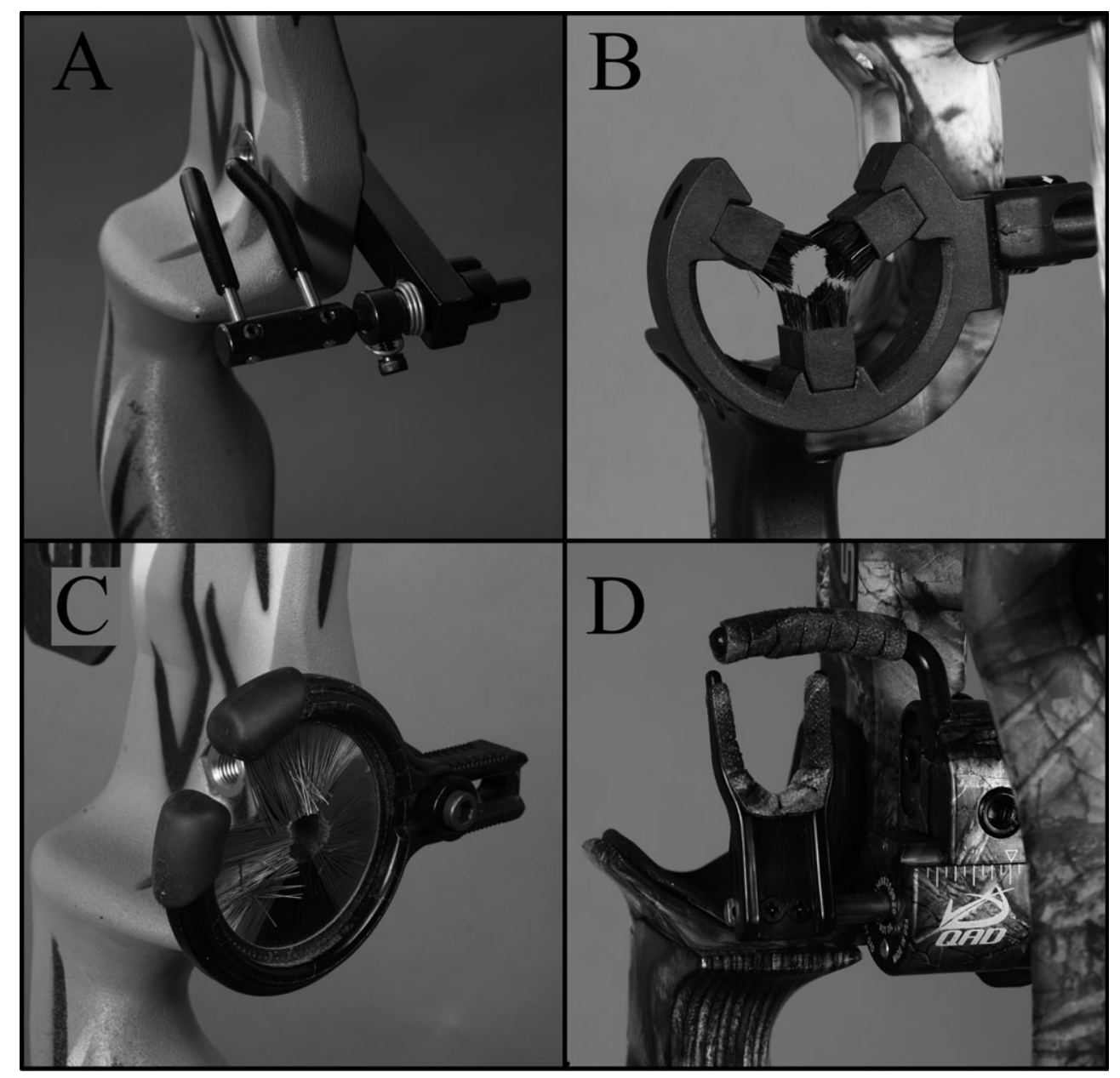

FIG. 11-Arrow rests: (A) shoot-through rest; (B) triple-point containment rest; (C) Trophy Ridge ${ }^{\circledR}$ Whisker Biscuit ${ }^{\circledR}$ rest; (D) drop-away rest.

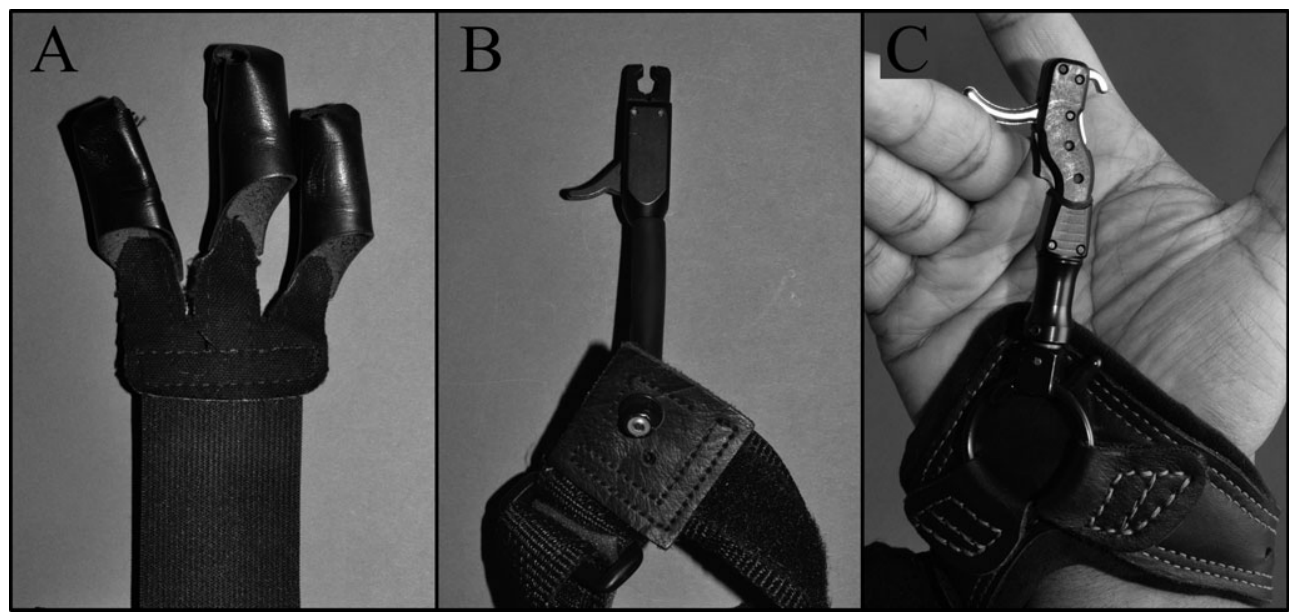

FIG. 12-Bowstring release aids: (A) shooting glove; (B) caliper release aid; (C) hook release aid.

the strain of the bowstring (Fig. 12A). The long risers and limbs of these bows require comparatively longer strings than the shorter risers and limbs of compound bows. The longer bowstring creates a larger angle between the nocked arrow and the string when the traditional bow is fully drawn, contrasted with the more acute angle created by the compound bow. This more acute angle produces a sharper pinch on the fingers if the archer uses them to draw the bow. To ease the drawing of the bow and to produce a steady hold on the bowstring that diminishes imprecision with its release, most compound bow shooters use "mechanical release aids." The release aid clips onto the string inferior to the arrow and nock or onto a string loop that straddles the top and bottom of the arrow nocking point. 
Various types of release aids saturate the archery market, and for hunting, there are two dominant styles of mechanical release aids: caliper releases and string-loop releases. Both styles of release aids fit entirely in the dorsum of the hand or are attached to a wrist strap. Caliper releases have two jaws that close around the bowstring and open by pressing a trigger (Fig. 12B). Stringloop mechanical release aids have a single hook or bar (Fig. 12C). This hook seizes a D-shaped string loop tied around the bowstring and is used to draw the bow back. A similar trigger relaxes the hook and releases the string.

Sight-Instinctive shooting, a school of thought held by some traditional bow archers where skill through the body's instincts overrides aiming and shooting, is very rarely employed by compound bow users. In fact, risers integrate a "sight window" immediately superior to the arrow shelf specifically to attach an external sight. The sight assists the archer in aiming the arrow toward the target and predominantly consists of a single pin or a series of pins. Omitting descriptions of specialty sights, two major types of bow sights are fixed-pin and movable-pin sights (11).

Fixed-Pin Sights-The fixed-pin sights commonly have metal or plastic pins housed within one or several vertical tracks. Each pin has vertically movement to indicate a specific distance. For example, a sight may have pins where the uppermost is accurate for shots taken at 20 yards, the middle pin adjusted for 30 yards, and the lowest pin adjusted for 40 yards. In addition to adjustments for vertical elevation, windage or lateral adjustments to the pins is necessary.

The process of "sighting" in the pins is straightforward but does require precision from the archer. The first step is to shoot two or more arrows at the target while aiming at the same point. The sight pin or entire sight pin housing is then moved toward the area where the arrows hit. As an example, if the arrows struck up and to the left of the aiming point, then the pins are moved superiorly and to the left lateral. The other pins are then fined tuned for the subsequent desired distances. If an archer needs to shoot at distances where pins are not set, they must "gap shoot" and aim using the space in between two pins flanking the desired distance. For example, if the archer needs to shoot a target at 35 yards but only has a 30 yard and 40 yard pin, then the space between the two pins represents the desired 35 yard distance.

Moveable-Pin Sights-Moveable-pin sights have 1-7 pins within the sight housing and the housing can move to make adjustments for distance at any time. The principle of these sights is that a wheel or knob integrated into the housing allows the archer to dial the sight pin or housing superiorly or inferiorly for increased or decreased shooting distances. Calibrating instructions are specific for the brand and model of the sight but routinely require affixing distance tapes or labels to the adjustment wheel to indicate calibrated distances. Once adjusted, the moveable-pin sights eliminate gap shooting by dialing to any distance within the sight's range.

Independent of the type, bow sights have common features and accessories. The first is the sight pin diameter. Four commonly used diameters are 0.010", 0.019", 0.029", and 0.040". Having separate sight pin diameters balances sight pin and target discernment when shooting at longer distances. At close distances, the target makes up more of the visual field and using a smaller or larger pin is of little consequence. At increased distances, the target's contribution to the visual field diminishes and larger pins obscure major portions of the target. This greatly

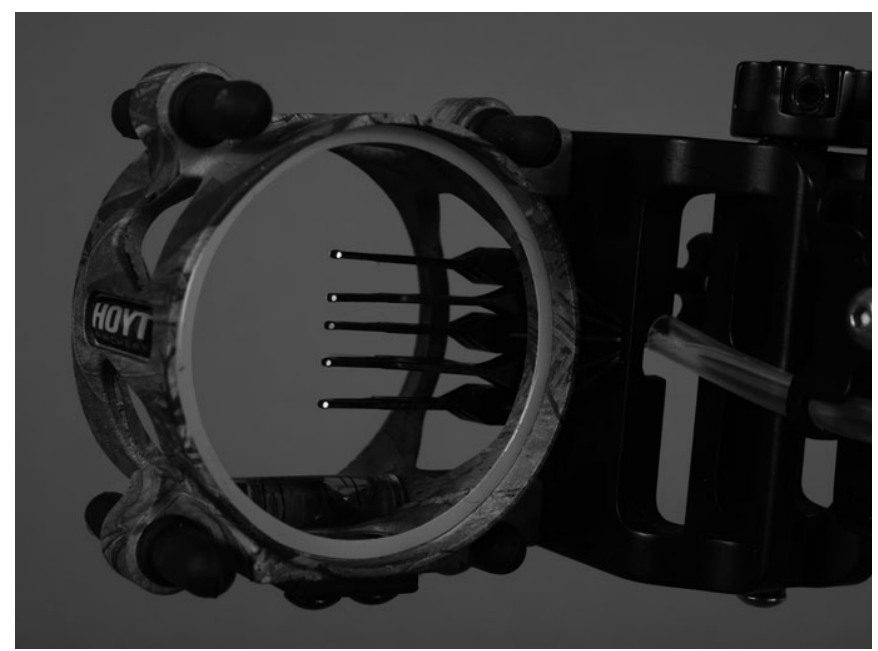

FIG. 13-Bow sight with fixed, fiber optic pins.

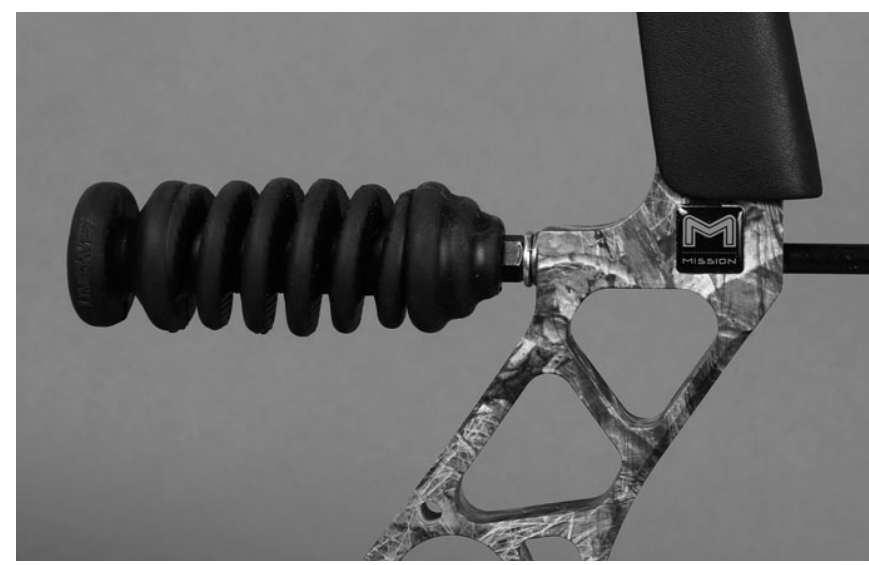

FIG. 14-Example of a rubberized stabilizer.

reduces precision shooting, but using very fine pins can be difficult for the archer to see. As a compromise, instead of having uniform diameter pins for all distances, some archers mount multiple sight pin diameters on a single sight pin housing.

Illumination of the sight pins is another modifiable feature and can be either nonelectric and/or electric. The most common nonelectric method is through the use of fiber optics (Fig. 13). Fiber optic threads extend a few inches to yards from the tip of the sight pin, and they point posterior or are wrapped around the sight pin housing. The fiber optic captures ambient light and directs it toward the exposed fiber end at the sight pin tip. In low light conditions, an attached LED light shines upon the fiber. There are even a few bow sights that utilize tritiumembedded sight pins for continuous illumination.

Stabilizer-Stabilizers are aptly named because the main function of these accessories is to stabilize the bow and allow for steady, smooth shooting of the bow. They thread into the riser and project anteriorly. Just as with all the other components of the bow, stabilizers have varied shapes and sizes. Commonalities to most all stabilizers are a cylindrical body, dampening material, and weight.

The proper length of the cylindrical body is dependent on the purpose of the bow, but follows a general rule that the length of 


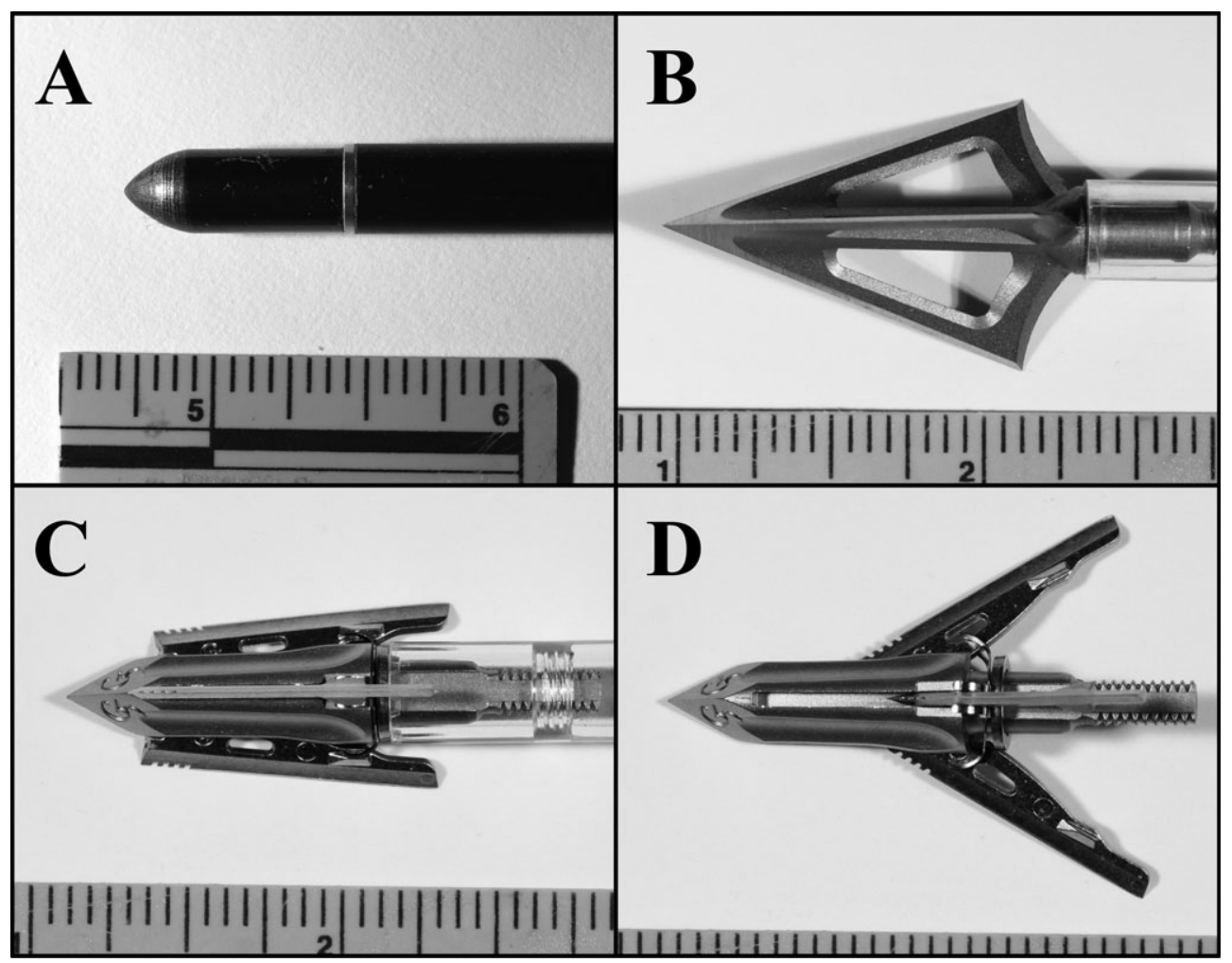

FIG. 15-Examples of arrowheads: (A) field point; (B) fixed-blade arrowhead; (C) mechanical arrowhead with blades retracted; (D) mechanical arrowhead with blades extended.

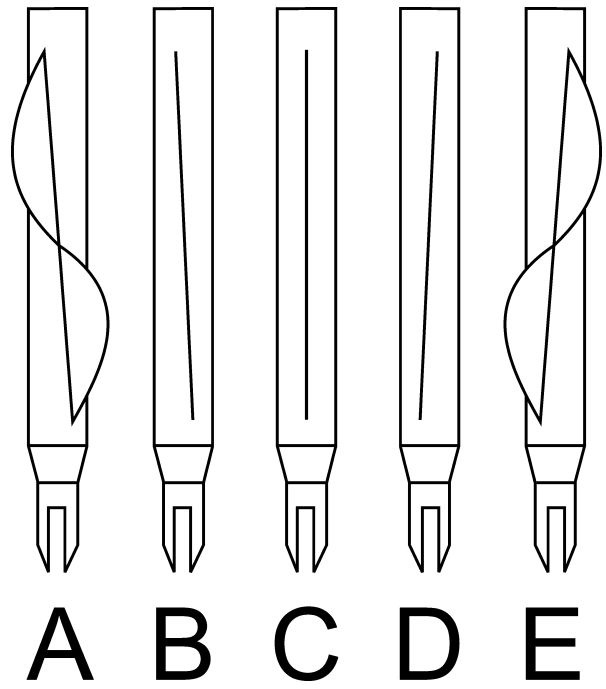

FIG. 16-Fletching orientation: (A) left helical; (B) left offset; (C) straight; (D) right offset; (E) right helical.

the stabilizer is directly related to the degree of stabilization provided. Target and competition archers frequently employ stabilizers ranging from 25 inches to 40 inches. Because these extreme lengths prohibit movement and increase the difficulty in masking of movement during hunting, stabilizers used in hunting are usually between 4 and 10 inches long.

The dampening material functions to reduce noise and vibrations generated from firing the bow. Even if the limbs are in parallel orientation, some vibrations are still generated. These vibrations travel from the limbs, into the riser, and along the

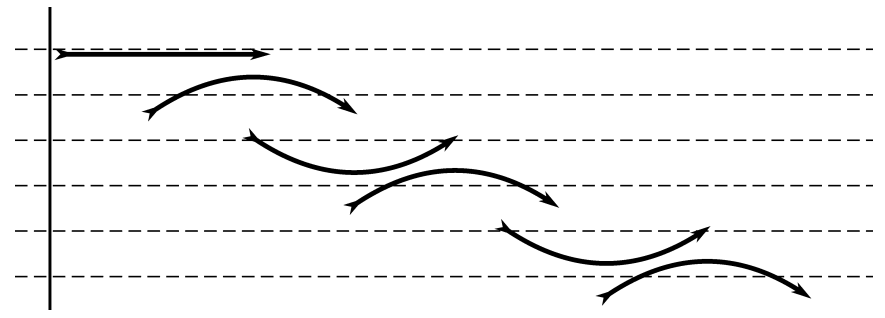

FIG. 17-Arrow oscillation during flight.

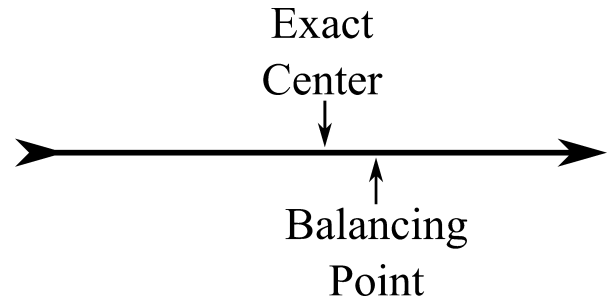

FIG. 18-Arrow balancing point comparison.

stabilizer. Rubber material placed on or near the end of the stabilizer assists in reducing these vibrations (Fig. 14).

Although it may seem counterintuitive that adding weight to a weapon helps to make it easier to shoot, stabilizers can do just that. The weight of the stabilizer, especially in those where the additional weights attach to the distal end of the cylindrical body, acts as a counterbalance to the weight of the bow and the drawing force directed posteriorly. 
Arrow-Technically, arrows are neither intrinsic nor extrinsic components of a bow, but without them, the weapon is useless. The arrow has seen much evolution from the early wooden ones paired with self bows to the modern ones used today.

Although a few compound bows can use wooden arrows, most arrow shafts are fiberglass, aluminum, carbon fiber, or carbon fiber with alloy cores. Fiberglass arrows generally display better arrow-to-arrow consistency and are frequently used for training purposes. However, their increased weight and fragile nature limit their use. Aluminum arrows are lighter than fiberglass ones and are popular with target shooters and beginner archers. They are inexpensive and are reusable even with minor damage. Carbon fiber arrows have smaller diameters than their aluminum arrow counterparts due to the strength of this material. Their light weight allows for higher velocities and for longer travel distances. The disadvantage of these arrows is that the carbon fiber can be fragile and is not subject to reuse after damage.

Two other main components of the arrow are the arrow tip and "fletching." Many arrows have threaded inserts glued into the distal end of the shaft, which allow affixing arrow tips into the arrows. Two dichotomies of arrow tips are field/practice tips and hunting tips. Field points are tapered to a point or have a bullet-shaped tip and are used strictly for target shooting (Fig. 15A). The hunting tips have a myriad of shapes and configurations. Small game hunting tips produce crush injuries through the use of blunted ends. Medium and large game hunting tips have attached razor-sharp blades to transect vessels and perforate soft tissue and organs. These tips, called broadheads, are fixed with immovable blades or are mechanical with blades that fold out or move to create larger wounds (Fig. 15B-D).

The fletching or vanes are paired with the arrow shafts to produce stability in flight and increases accuracy. They are usually made from natural feathers or polymer plastics and vary in size and placement on the arrow shaft. Three to four glued vanes are equally spaced on the proximal end of the arrow shaft. They have one of five arrangements: left helical, left offset, straight, right offset, and right helical (Fig. 16A-E). The most clear-cut are straight vanes, where the fletching is parallel to the arrow shaft. This placement provides the greatest arrow velocities but also exhibit the greatest loss of energy at increased distances. Helical fletching have a spiral pattern while offset vanes are glued linearly on the shaft but have a slight turn. Both of these orientations can be directed right or left, and although air resistance causes all fletched arrows to rotate regardless of style, helical and offset vanes induce greater rotational stability of the arrow than the straight vanes.

An occurrence that is ubiquitous to all arrows upon release is flexing of the shaft as it is accelerated toward the target. Although a single unit, the greater mass of the arrow tip at the distal end of the arrow more greatly resists acceleration compared to the much lighter nock at the proximal end, causing oscillation of the arrow in a plane perpendicular to the bowstring (Fig. 17). The maximum peak-to-peak amplitude of the arrow's oscillations represents the greatest variation from the point of aim, or line of sight to the target. The vanes help attenuate the arrow's oscillation and attenuate the peak-to-peak amplitude.

The rotation of the arrow created by the fletching moves the single plane oscillations to a $360^{\circ}$ circle around the point of aim. Additionally, flexion of the arrow does not occur around the exact center of the arrow shaft, but at its balancing point, also known as the "Forward of Center" (FOC). Due to the greater mass of the arrow tip, the FOC is closer toward the tip than the nock (Fig. 18). Combining the FOC with the dampening of oscillations and rotation of the arrow created by the fletching, a smaller variation from the point of aim is created and increased accuracy results.

\section{Conclusion}

Archery for survival and sport is engrained in human culture, and much technological evolution has occurred in the past halfcentury. However, due to the extensive supply of firearms, knowledge of the modern compound bow and arrow injuries by the forensic pathologist is understandably lacking. This study provides an overview of the modern compound bow to familiarize forensic scientists with this form of weaponry.

\section{References}

1. Lombard M, Haidle M. Thinking a bow-and-arrow set: cognitive implications of middle stone age bow and stone-tipped arrow technology. Camb Archaeol J 2012;22(2):237-64.

2. Lombard M, Phillipson L. Indications of bow and stone-tipped arrow use 64000 years ago in KwaZulu-Natal, South Africa. Antiquity 2010;84 (325):635-48.

3. McEwen E, Miller R, Bergman C. Early bow design and construction. Sci Am 1991;246(6):76-82.

4. Kooi BW. The design of the bow. Proceedings Koninklijke Mederlandse Akademie van Wetenschappen 1994;97(3):1-27.

5. Koo BW. On the mechanics of the modern working-recurve bow. Comput Mech 1991;8(5):291-304.

6. Holless Wilbur Allen 1909-1979. http://archeryhalloffame.com/Allen.html (accessed September 14, 2015).

7. Maker builds a 3D printed compound bow that can shoot at 10 meters. http://www.3ders.org/articles/20141122-maker-builds-a-3d-printed-com pound-bow-that-can-shoot-at-10-meters.html (accessed September 14, 2015).

8. Wise L. Selecting correct bow length. Arrow Trade Magazine 2008;12 (6):78-84.

9. Wise L. Understanding force draw curves. Arrow Trade Magazine 2006;10(6):78-83.

10. Easton Archery - Bowstring Tech Tips. http://www.eastonarchery.com/ blog/bowstring-tech-tips (accessed October 30, 2014).

11. Compound Bow Sight Selection. http://www.huntersfriend.com/sight selection_guide/sight_selection_guide.html (accessed November 1, 2014).

Additional information and reprint requests:

LokMan Sung, M.D.

Department of Pathology

University of Michigan

4232 Medical Science I1301 Catherine Street

Ann Arbor

MI 48109

E-mail: Lsung@waynecounty.com 